\title{
Numerical and exact solutions for time fractional Burgers' equation
}

\author{
Asıf Yokuş ${ }^{a, *}$, Doğan Kaya ${ }^{b}$ \\ ${ }^{a}$ Firat University, Department of Actuary, Elazig, Turkey. \\ ${ }^{b}$ Istanbul Commerce University, Department of Mathematics, Istanbul, Turkey. \\ Communicated by Y. $-\mathrm{Z}$. Chen
}

\begin{abstract}
The main purpose of this paper is to find an exact solution of the traveling wave equation of a nonlinear time fractional Burgers' equation using the expansion method and the Cole-Hopf transformation. For this purpose, a nonlinear time fractional Burgers' equation with the initial conditions considered. The finite difference method (FDM for short) which is based on the Caputo formula is used and some fractional differentials are introduced. The Burgers' equation is linearized by using the ColeHopf transformation for a stability of the FDM. It shows that the FDM is stable for the usage of the Fourier-Von Neumann technique. Accuracy of the method is analyzed in terms of the errors in $\mathrm{L}_{2}$ and $\mathrm{L}_{\infty}$. All of obtained results are discussed with an example of the Burgers' equation including numerical solutions for different situations of the fractional order and the behavior of potentials $u$ is investigated with graphically. All the obtained numerical results in this study are presented in tables. We used the Mathematica software package in performing this numerical study. (C)2017 All rights reserved.
\end{abstract}

Keywords: Nonlinear time fractional Burgers' equation, an expansion method, finite difference method, Caputo formula, linear stability, Cole-Hopf transformation.

2010 MSC: 65M06, 35R11.

\section{Introduction}

In the recent years, several works have been done in the construction of the approximate solutions for various nonlinear fractional partial differential equations (nfPDE for short) $[13,15,17,26]$. In some senses linear and nonlinear fractional differential equations can be used to represent various real-life problems. Therefore, developing methods for finding the exact solutions to these types of equations is very important. Studies in finding exact solutions to nfPDE, when they exist, are very important for the understanding of most fractional nonlinear physical phenomena. However, it is not easy to solve some forms of nfPDE. In order to solve over these problems, we need to have a variable transformation on the fractional equation. In this case, nfPDE will be in form of integer-order differential equations. Actually, there are some kinds of fractional derivatives and some useful formulas in literature $[1,6,10-12,18$ $21,25,28]$ for these types of transformations. In this paper, we use the classical Cole-Hopf transformation [6] in order to obtain exact solution of a time fractional Burgers' equation.

\footnotetext{
${ }^{*}$ Corresponding author

Email addresses: asf yokus@firat.edu.tr (Asıf Yokuş), dogank@ticaret.edu.tr (Doğan Kaya)
} 
We present an expansion method by inspiring the $\left(\mathrm{G}^{\prime} / \mathrm{G}\right)$-expansion method which was introduced by Wang et al. [22] and the other similar methods [7, 24]. We implement this method to find several analytic solutions of the time fractional Burgers' equation in form of rational trigonometric solutions, but we consider one of these solutions for the numerical examination. However, there are many methods for obtaining traveling wave solutions of the nonlinear partial differential equations (nPDE for short) in literature $[3-5,16,23,27]$.

In this study, we use an analytical method and numerical method to treat time fractional Burgers' equation. In order to find exact solution, we use the $\left(\mathrm{G}^{\prime} / \mathrm{G}\right)$-expansion method and the Cole-Hopf transformation. We show that nfPDE can be converted to $\mathrm{nPDE}$ or linear ordinary differential equation (IODE for short) form by using simple transformation of the variables considered nfPDE. The process is wellknown whereby the advanced calculus can be dealt with fractional calculus without any difficulty. Doing this by the starting point, applicability of the method can be used effectively.

To show the effectiveness of these exact and numerical methods, we solve the time fractional Burgers' equation of the form [8]

$$
\frac{\partial^{\alpha} u(x, t)}{\partial t^{\alpha}}+u(x, t) \frac{\partial u(x, t)}{\partial x}=\delta \frac{\partial^{2} u(x, t)}{\partial x^{2}}
$$

where $0<\alpha \leqslant 1$ and $\delta$ is a constant. Equation (1.1) uses a Caputo fractional derivative of order $\alpha$, defined by

$$
\frac{\partial^{\alpha} f(x, t)}{\partial t^{\alpha}}=\frac{1}{\Gamma(\beta-\alpha)} \int_{0}^{t} \frac{\frac{\partial^{\beta}}{\partial \xi^{\beta}} f(x, \xi)}{(t-\xi)^{\alpha-\beta+1}} d \xi,
$$

where $\beta$ is an integer that $\beta-1<\alpha \leqslant \beta$. The function $\Gamma$ (.) is called as a Gamma function.

\section{Analysis of $\left(1 / G^{\prime}\right)$-expansion method}

Consider the two-variable general form of nPDE

$$
\mathrm{Q}\left(\mathrm{u}, \frac{\partial^{\alpha} \mathrm{u}}{\partial \mathrm{t}^{\alpha}}, \frac{\partial \mathrm{u}}{\partial x}, \frac{\partial^{2} \mathrm{u}}{\partial x^{2}}, \ldots\right)=0,
$$

and transform Eq. (1.1) with $u=u(x, t)=u(\xi), \xi=x-\frac{V t^{\alpha}}{\Gamma(1+\alpha)}$, where $V$ is a constant. Doing this, we obtain the following nonlinear ODE for $u(\xi)$ :

$$
\mathrm{Q}^{\prime}\left(\mathrm{u}^{\prime}, \mathrm{u}^{\prime \prime}, \mathrm{u}^{\prime \prime \prime}, \ldots\right)=0 \text {. }
$$

The solution of Eq. (2.2) is considered to be in the following form:

$$
u(\xi)=a_{0}+\sum_{i=1}^{m} a_{i}\left(\frac{1}{G^{\prime}}\right)^{i},
$$

where $a_{i}$ are constants, $m$ is a positive integer which will be determined by balancing the highest order derivative and with the highest power nonlinear terms in Eq. (2.2), and $G=G(\xi)$ satisfies the following second order lODE

$$
\mathrm{G}^{\prime \prime}+\lambda \mathrm{G}^{\prime}+\mu=0,
$$

where $\mu$ and $\lambda$ are constants to be determined later.

Substituting Eq. (2.3) and its possible derivatives into Eq. (2.2) and simplifying along with the Eq. (2.4), yields a polynomial in $\left(1 / \mathrm{G}^{\prime}\right)$. We find a set of algebraic equations by setting the coefficients of $\left(1 / G^{\prime}\right)$ that have the same order to zero. We simplify these algebraic equations to find $a_{i}, i \geqslant 0$ and $V$ constants and then substituting $a_{i}$ and the general solutions of equation (2.4) into (2.3), we can obtain solutions of the equation (2.1). 


\section{Analysis of finite difference method}

We will need some notations to describe the finite forward difference method. $\Delta x$ is the spatial step, $\Delta t$ is the time step, $x_{i}=a+i \Delta x, i=0,1,2, \ldots, N$ points are the coordinates of mesh and $N=\frac{b-a}{\Delta x}, t_{j}=$ $j \Delta t, j=0,1,2, \ldots, M$ and $M=\frac{T}{\Delta t}$. The function $u(x, t)$ is the values of the solution at these grid points which are $u\left(x_{i}, t_{j}\right) \cong u_{i, j}$, where $u_{i, j}$ represent the numerical solutions of the exact value of $u(x, t)$ at the point $\left(x_{i}, t_{j}\right)$. We write the difference operators as

$$
\begin{aligned}
H_{t} u_{i, j} & =u_{i, j+1}-u_{i, j}, \\
H_{x} u_{i, j} & =u_{i+1, j}-u_{i, j} \\
H_{x x} u_{i, j} & =u_{i+1, j}-2 u_{i, j}+u_{i-1, j} .
\end{aligned}
$$

Thus partial derivatives are approximated through the finite difference operators as

$$
\begin{gathered}
\left.\frac{\partial u}{\partial x}\right|_{i, j}=\frac{H_{x} u_{i, j}}{\Delta x}+O\left(\Delta x^{2}\right), \\
\left.\frac{\partial^{2} u}{\partial x^{2}}\right|_{i, j}=\frac{H_{x x} u_{i, j}}{(\Delta x)^{2}}+O\left(\Delta x^{2}\right) .
\end{gathered}
$$

According to the shifted Caputo definition [2],

$$
\frac{\partial^{\alpha} u(x, t)}{\partial t^{\alpha}} \cong \begin{cases}\frac{h^{-\alpha}}{\Gamma(2-\beta)} H_{t} u+\frac{h^{-\alpha}}{\Gamma(2-\beta)} \sum_{k=1}^{i} H_{t} u_{i, j-k} f(k), & j \geqslant 1 \\ \frac{h^{-\alpha}}{\Gamma(2-\beta)} H_{t} u_{i, 0}, j & =0 .\end{cases}
$$

There are many studies in the literature on fractional derivatives of Taylor series. One of these studies has been awarded the generalized Taylor series by Odibat and Shawagfeh [14]

$$
\left.\frac{\partial^{\alpha} u(x, t)}{\partial t^{\alpha}}\right|_{i, j}=\Gamma(\alpha+1)(\Delta t)^{-\beta} H_{t} u_{i, j}+O\left(\Delta t^{2 \alpha}\right) .
$$

In the finite difference method, substituting Eqs. (3.2), (3.3), and (3.4) into Eq. (1.1), we get the following indexed form

$$
u_{i+1, j}=\frac{-\left(\delta u_{i-1, j}+u_{i, j}\left(-2 \delta+\Delta x u_{i, j}\right)\right)+\vartheta\left[H_{t} u_{i, j}-\sum_{k=1}^{j} f(k)\left(H_{t} u_{i, j-k}\right)\right]}{\delta-\Delta x u_{i, j}},
$$

where $\vartheta=\frac{(\Delta x)^{2}}{(\Delta t)^{\alpha} \Gamma(2-\alpha)}, f(k)=-k^{1-\alpha}+(1+k)^{1-\alpha}$ and the initial values $u_{i, 0}=u_{0}\left(x_{i}\right)$.

\section{Consistency analysis}

In this section, we investigate the consistency of Eq. (1.1) by finite difference method. At first, the Taylor series expansions can be given in the following form,

$$
\begin{aligned}
& u_{i+1, j}=u_{i, j}+\Delta x \frac{\partial u}{\partial x}+(\Delta x)^{2} \frac{\partial^{2} u}{\partial x^{2}}+O\left(\Delta x^{3}\right), \\
& u_{i, j+1}=u_{i, j}+\Delta t \frac{\partial u}{\partial t}+(\Delta t)^{2} \frac{\partial^{2} u}{\partial t^{2}}+O\left(\Delta t^{3}\right), \\
& u_{i-1, j}=u_{i, j}-\Delta x \frac{\partial u}{\partial x}+(\Delta x)^{2} \frac{\partial^{2} u}{\partial x^{2}}-O\left(\Delta x^{3}\right) .
\end{aligned}
$$


Let us define an operator $L$ by

$$
L=\frac{\partial}{\partial t}+u \frac{\partial}{\partial x}-\delta \frac{\partial^{2}}{\partial x^{2}}
$$

and the indexed form of operator L can be written as

$$
L_{i, j}=\frac{H_{t} u_{i, j}}{\Delta t}+u \frac{H_{x} u_{i, j}}{\Delta x}-\delta \frac{H_{x x} u_{i, j}}{(\Delta x)^{2}} .
$$

If we substitute the indexed form (4.1a), (4.1b), and (4.1c) into the equality (4.2) and do some necessary manipulations, then the approach will be $\Delta t \rightarrow 0$ and $\Delta x \rightarrow 0$. Hence the equality (4.2) will be same as left hand side of the Eq. (1.1). This conclusion shows that Eq. (1.1) is consistent by finite difference method.

\section{Truncation error and stability analysis}

In this section, we mainly study the stability and error analysis for the finite forward difference method. With the stability, we discuss if there is a perturbation in the initial condition and then the small change will not cause the large error in the numerical solution. Simply stability means that the scheme does not amplify errors and the error caused by a small perturbation in the numerical solution remains bound.

Theorem 5.1. The truncation error of the finite different method to the Burgers' equation (1.1) is $\mathrm{O}\left((\Delta \mathrm{t})^{2 \alpha}+\right.$ $\left.(\Delta x)^{2}\right)$.

Proof. Substituting Eqs. (3.2), (3.3), and (3.4) into Eq. (1.1) gives

$$
\begin{aligned}
\frac{h^{-\alpha}}{\Gamma(2-\beta)} H_{t} u & +\frac{h^{-\alpha}}{\Gamma(2-\beta)} \sum_{k=1}^{i} H_{t} u_{i, j-k} f(k)+O\left(\Delta t^{2 \alpha}\right) \\
& +u_{i, j}\left(\frac{H_{x} u_{i, j}}{\Delta x}+O\left(\Delta x^{2}\right)\right)-\delta\left(\frac{H_{x x} u_{i, j}}{(\Delta x)^{2}}+O\left(\Delta x^{2}\right)\right)=0 .
\end{aligned}
$$

Eq. (5.1) can be rearranged in the following form after some algebraic manipulations,

$$
\frac{h^{-\alpha}}{\Gamma(2-\beta)} H_{t} u+\frac{h^{-\alpha}}{\Gamma(2-\beta)} \sum_{k=1}^{i} H_{t} u_{i, j-k} f(k)+u_{i, j} \frac{H_{x} u_{i, j}}{\Delta x}-\delta \frac{H_{x x} u_{i, j}}{(\Delta x)^{2}}+O\left(\Delta t^{2 \alpha}+\Delta x^{2}\right)=0 .
$$

If we separate the truncation error $\mathrm{O}\left(\Delta \mathrm{t}^{2 \alpha}+\Delta \mathrm{x}^{2}\right)$ term from Eq. (5.2), the rest of the equation will be the indexed of the Burgers' equation (1.1) as

$$
\frac{h^{-\alpha}}{\Gamma(2-\beta)} H_{t} u+\frac{h^{-\alpha}}{\Gamma(2-\beta)} \sum_{k=1}^{i} H_{t} u_{i, j-k} f(k)+u_{i, j} \frac{H_{x} u_{i, j}}{\Delta x}-\delta \frac{H_{x x} u_{i, j}}{(\Delta x)^{2}}=0 .
$$

If we substitute the equalities (3.1a)-(3.1c) into the Eq. (5.1) and do some necessary manipulations, then we obtain the following equality

$$
\begin{aligned}
u_{i+1, j}= & \frac{\vartheta\left[H_{t} u_{i, j}-\sum_{k=1}^{j} f(k)\left(H_{t} u_{i, j-k}\right)\right]-\delta u_{i-1, j}+u_{i, j}\left(2 \delta-\Delta x u_{i, j}\right)}{\delta-\Delta x u_{i, j}} \\
& +\frac{u_{i, j} \Delta x^{2} O\left(\Delta x^{2}\right)+\Delta x^{2}\left(O\left(\Delta t^{2 \alpha}\right)-\delta O\left(\Delta x^{2}\right)\right)}{\delta-\Delta x u_{i, j}} .
\end{aligned}
$$


By using equality (5.3), we can write numerical solution $\hat{U}$ as

$$
\hat{u}=\frac{\vartheta\left[H_{t} u_{i, j}-\sum_{k=1}^{j} f(k)\left(H_{t} u_{i, j-k}\right)\right]-\delta u_{i-1, j}+u_{i, j}\left(2 \delta-\Delta x u_{i, j}\right)}{\delta-\Delta x u_{i, j}},
$$

and the transaction error $E$ as

$$
E=\frac{u_{i, j} \Delta x^{2} O\left(\Delta x^{2}\right)+\Delta x^{2}\left(O\left(\Delta t^{2 \alpha}\right)-\delta O\left(\Delta x^{2}\right)\right)}{\delta-\Delta x u_{i, j}} .
$$

In addition to this, a transaction error $E$ can be written as

$$
\mathrm{E}=|\mathrm{U}-\hat{\mathrm{U}}|,
$$

where $\mathrm{U}$ is the exact solution. Moreover, considering Theorem 5.1, if $\Delta \mathrm{t}$ and $\Delta \mathrm{x}$ are chosen as small as necessary, transaction error will obviously be very small. What is more, limit of $E$ can be written as

$$
\lim _{\substack{\Delta x \rightarrow 0 \\ \Delta t \rightarrow 0}} E=0 .
$$

Here, if $\Delta \mathrm{t}$ and $\Delta \mathrm{x}$ are configured for a value close to zero $\varepsilon>0$, the following inequality can be given by

$$
|\mathrm{E}|<\varepsilon \text {. }
$$

This also shows that finite difference method is stable.

\section{Linear stability analysis}

Consider Burgers' equation (1.1) with the initial values $u(x, 0)=u_{0}$, recognized in domain of fractional Burgers' equation and boundary conditions of a simple type

$$
u\left(x_{1}, t\right)=0, u\left(x_{2}, t\right)=0,
$$

where $t>0$ and $x_{1} \leqslant x \leqslant x_{2}$. By using classical Cole-Hopf transformation [6], is given by

$$
u(x, t)=\psi_{x},
$$

where $\psi=\psi(x, t)$ and $\delta>0$. Substituting Eq. (6.1) into Eq. (1.1) gives,

$$
\frac{\partial^{\alpha} \psi_{x}}{\partial t^{\alpha}}+\psi_{x}\left(\psi_{x}\right)_{x}-\delta\left(\psi_{x}\right)_{x x}=0
$$

If we integrate Eq. (6.2) with respect to $x$ and ignore the functional integration we derive

$$
\frac{\partial^{\alpha} \psi}{\partial t^{\alpha}}+\frac{1}{2} \psi_{x}^{2}-\delta \psi_{x x}=0
$$

for the Cole-Hopf transformation, we have

$$
\begin{gathered}
\psi=-2 \delta \ln \phi \\
u(x, t)=\psi_{x}=-2 \delta \frac{\phi_{x}}{\phi},
\end{gathered}
$$

substituting Eq. (6.4) and (6.5) into non-linear equation (6.3) yields,

$$
\frac{\partial^{\alpha} \phi}{\partial t^{\alpha}}-\delta \phi_{x x}=0
$$

Eq. (6.6) is known as a heat flow equation which may be written in the finite difference operator form as:

$$
\frac{\partial^{\alpha} \phi}{\partial t^{\alpha}}-\delta \frac{H_{x x} \phi_{i, j}}{\Delta x^{2}}=0
$$


Theorem 6.1. The finite difference method for the Burgers' equation is unconditionally linear stable.

Proof. We consider the Von Neumann's stability of the finite difference method for the Burgers' equation. Let

$$
u_{i, j}=u(i \Delta x, j \Delta t)=u(p, q)=\varepsilon^{q} e^{I \xi p}, \quad \xi \in[-\pi, \pi],
$$

where $p=i \Delta x, q=j \Delta t$ and $I=\sqrt{-1}$. If we substitute the equalities (3.1c), (3.4), and (6.8) into the equality (6.7) we obtain

$$
\varepsilon=1-\frac{2 \delta \Delta t(1-\operatorname{Cos} \xi)}{(\Delta x)^{2}}
$$

where $\alpha=1$. According to the Von Neumann's stability analysis, if $|\varepsilon| \leqslant 1$, the finite difference methods for the Burgers' equation is stable. For special choice of $\Delta x=\Delta t$, the following inequality can be written as

$$
|\varepsilon| \leqslant 1 \Leftrightarrow|\Delta x|=-2(-\delta+\operatorname{Cos} \xi) .
$$

\section{7. $\mathrm{L}_{2}$ and $\mathrm{L}_{\infty}$ error norms}

For the test problem used in the present study, numerical results of the equation have been obtained and all calculations in this study have been done by using the Mathematica program. To show that how much the analytical results and numerical results are close to each other we use $L_{2}$ and $L_{\infty}$ error norms. The $L_{2}$ error norm is defined as [9]

$$
\mathrm{L}_{2}=\left\|\mathrm{u}^{\text {exact }}-\mathrm{u}^{\text {numeric }}\right\|_{2}=\sqrt{h \sum_{j=0}^{\mathrm{N}}\left|\mathrm{u}_{j}^{\text {exact }}-\mathrm{u}_{j}^{\text {numeric }}\right|^{2}},
$$

and $\mathrm{L}_{\infty}$ error norm is defined as

$$
\mathrm{L}_{\infty}=\left\|\mathrm{u}^{\text {exact }}-\mathrm{u}^{\text {numeric }}\right\|_{\infty}=\operatorname{Max}_{j}\left|\mathrm{u}_{\mathrm{j}}^{\text {exact }}-\mathrm{u}_{\mathrm{j}}^{\text {numeric }}\right| .
$$

\section{Implementation of the methods}

\subsection{Expansion method}

Let us consider Burgers' equation (1.1). Thus, after using transformation $u=u(x, t)=u(\xi), \xi=$ $x-\frac{\mathrm{Vt}^{\alpha}}{\Gamma(1+\alpha)}$, taking once the integral of Eq. (1.1) we get

$$
c-V u+\frac{1}{2} u^{2}-\delta u^{\prime}=0,
$$

where $c$ is a constant of integration. Then balancing $u^{2}$ with $u^{\prime}$ gives $m=1$. Therefore, we may choose

$$
u(\xi)=a_{0}+a_{1}\left(\frac{1}{G^{\prime}}\right) \text {. }
$$

Substituting Eq. (8.1) into (8.2) yields a group of algebraic equations for the coefficients $a_{0}, a_{1}, \delta, \mu, c, \lambda$, and $V$. These systems are

$$
\begin{aligned}
& \left(\frac{1}{G^{\prime}}\right)^{0}: \frac{a_{0}^{2}}{2}+c-a_{0} V=0 \\
& \left(\frac{1}{G^{\prime}}\right)^{1}: a_{1}\left(a_{0}-V-\delta \lambda\right)=0 \\
& \left(\frac{1}{G^{\prime}}\right)^{2}: \frac{a_{1}^{2}}{2}-a_{1} \delta \mu=0
\end{aligned}
$$

Finding the solutions of system (8.3) with the aid of Mathematica, we obtained the following cases. 
Case 1. If

$$
a_{0}=\delta \lambda-\sqrt{2 c+\delta^{2} \lambda^{2}}, \quad a_{1}=2 \delta \mu, \quad V=-\sqrt{2 c+\delta^{2} \lambda^{2}}
$$

and substitute values found (8.4) into (8.2), we have two types of wave solutions of Eq. (1.1):

$$
\begin{gathered}
\xi=x+\frac{\left(\sqrt{2 c+\delta^{2} \lambda^{4}}\right) t^{\alpha}}{\Gamma(1+\alpha)}, \\
u_{1}(\xi)=\delta \lambda-\sqrt{2 c+\delta^{2} \lambda^{2}}+2 \delta \mu\left(\frac{1}{-\frac{\mu}{\lambda}+A(\operatorname{Cosh}(\xi \lambda)-\operatorname{Sinh}(\xi \lambda))}\right) .
\end{gathered}
$$

Case 2. If

$$
a_{0}=\delta \lambda+\sqrt{2 c+\delta^{2} \lambda^{2}}, \quad a_{1}=2 \delta \mu, \quad V=\sqrt{2 c+\delta^{2} \lambda^{2}},
$$

and substitute (8.6) into (8.2), we have three two of traveling wave solutions of Eq. (1.1):

$$
\begin{gathered}
\xi=x-\frac{\left(\sqrt{2 c+\delta^{2} \lambda^{4}}\right) t^{\alpha}}{\Gamma(1+\alpha)}, \\
u_{2}(\xi)=\delta \lambda+\sqrt{2 c+\delta^{2} \lambda^{2}}+2 \delta \mu\left(\frac{1}{-\frac{\mu}{\lambda}+A(\operatorname{Cosh}(\xi \lambda)-\operatorname{Sinh}(\xi \lambda))}\right) .
\end{gathered}
$$

\subsection{Cole-Hopf transformation}

By using the transformation

$$
\phi(x, t)=\Phi(\zeta), \quad \zeta=x-\frac{V^{\alpha}}{\Gamma(1+\alpha)}
$$

on Eq. (6.6), where $V$ is nonzero arbitrary constant, (6.6) can be rewritten in the following lODE form

$$
-\mathrm{V} \Phi^{\prime}-\delta \Phi^{\prime \prime}=0,
$$

integrating Eq. (8.8) with respect to $\zeta$, implies

$$
c-V \Phi-\delta \Phi^{\prime}=0,
$$

where $c$ is a constant and traveling wave solution of Eq. (8.9) is

$$
\Phi(\zeta)=\frac{c}{V}+A e^{-\frac{V \zeta}{\delta}}
$$

The solution of this equation can be written by use of the Cole-Hopf transformation inverse of Eq. (8.10)

$$
u(x, t)=\frac{2 A V^{2}}{A V+c\left[\operatorname{Cosh}\left(\frac{V}{\delta}\left(x-\frac{V t^{\alpha}}{\Gamma(1+\alpha)}\right)\right)+\operatorname{Sinh}\left(\frac{V}{\delta}\left(x-\frac{V t^{\alpha}}{\Gamma(1+\alpha)}\right)\right)\right]}
$$

The solution (8.11) is similar to (8.5) and (8.7) solutions.

\subsection{Finite difference method}

In this section, we consider numerical experiments that we have obtained by using the data as follows: $A=5, \delta=1, c=0, \lambda=\mu=0.5, \alpha=0.8,0<x<1$, and $0<t<1$ for Eq. (8.5), the initial condition is

$$
\begin{aligned}
& u_{0}(x)=u(x, 0)=\frac{1}{-1+5\left(\operatorname{Cosh} \frac{x}{2}-\operatorname{Sinh} \frac{x}{2}\right)}, \\
& u_{0}(x)=u(x, 0)=\frac{1}{-1+5\left(\operatorname{Cosh} \frac{x}{2}-\operatorname{Sinh} \frac{x}{2}\right)^{\prime}}
\end{aligned}
$$

and under the above assumptions the exact solution of the Eq. (1.1) is as follows 


$$
u(x, t)=\frac{1}{-1+5\left[\operatorname{Cosh}\left(\frac{x}{2}+\frac{t^{\alpha}}{4 \Gamma(1+\alpha)}\right)-\operatorname{Sinh}\left(\frac{x}{2}+\frac{t^{\alpha}}{4 \Gamma(1+\alpha)}\right)\right]} .
$$

A comparison of the obtained exact and numerical solutions are tabulated in Tables 1 and 2.

\begin{tabular}{ccccc}
$x_{i}$ & $t_{j}$ & Numerical solution & Exact solution & Error \\
\hline \hline 0.00 & 0.02 & 0.253126 & 0.2537010 & $5.75510 \times 10^{-4}$ \\
0.02 & 0.02 & 0.256321 & 0.2569060 & $5.84566 \times 10^{-4}$ \\
0.04 & 0.02 & 0.259566 & 0.2601590 & $5.93809 \times 10^{-4}$ \\
0.06 & 0.02 & 0.262860 & 0.2634630 & $6.03243 \times 10^{-4}$ \\
0.08 & 0.02 & 0.266204 & 0.2668170 & $6.12874 \times 10^{-4}$ \\
0.10 & 0.02 & 0.269601 & 0.2702230 & $6.22707 \times 10^{-4}$ \\
0.12 & 0.02 & 0.273050 & 0.2736830 & $6.32749 \times 10^{-4}$ \\
\hline
\end{tabular}

Table 1: Numerical and exact solutions of equation (1.1) and absolute errors when $\Delta x=0.02$.

\begin{tabular}{ccc}
$\Delta x=\Delta \mathrm{t}$ & $\mathrm{L}_{2}$ & $\mathrm{~L}_{\infty}$ \\
\hline \hline 0.20 & 0.0425678 & 0.070345 \\
0.10 & 0.0042708 & 0.006320 \\
0.05 & 0.0011366 & 0.001930 \\
0.04 & 0.0007889 & 0.001441 \\
0.03 & 0.0005043 & 0.001024 \\
0.02 & 0.0002769 & 0.000664 \\
0.01 & 0.0001045 & 0.000342 \\
\hline
\end{tabular}

Table 2: $\mathrm{L}_{2}$ and $\mathrm{L}_{\infty}$ error norm when $0 \leqslant \mathrm{~h} \leqslant 1$ and $0 \leqslant x \leqslant 1$.

From Tables 1 and 2 it is easily seen that results are in good agreement with the exact solution.
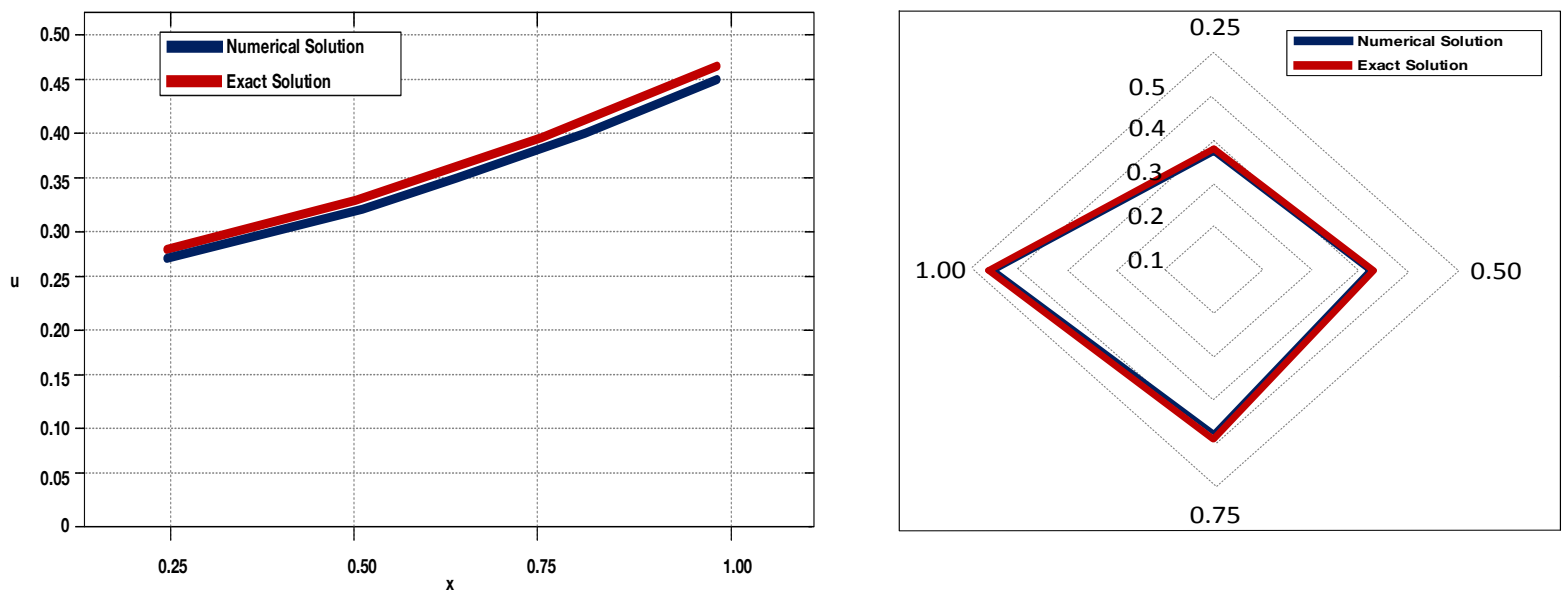

Figure 1: Numerical solution of equation (1.1) for finite difference and an expansion method.

Figure 1 demonstrates the physical behavior of the solution, and depicts that exact solution values are almost close to the numerically calculated values. We know that truncation error is due to the choice of $\Delta x$ and $\Delta t$. There are appointed close to zero indicates that the truncation error is very small. This resulting behavior of the numerical and exact solutions can be seen in the following graph by using values of $\Delta x=0.25$. 
Considering the Eq. (3.5) which is obtained by using finite difference method, we can say that the term of Caputo will increase when $\alpha$ decreases. It means that potential $u$ is increasing. We can see this situation in Figure 2.
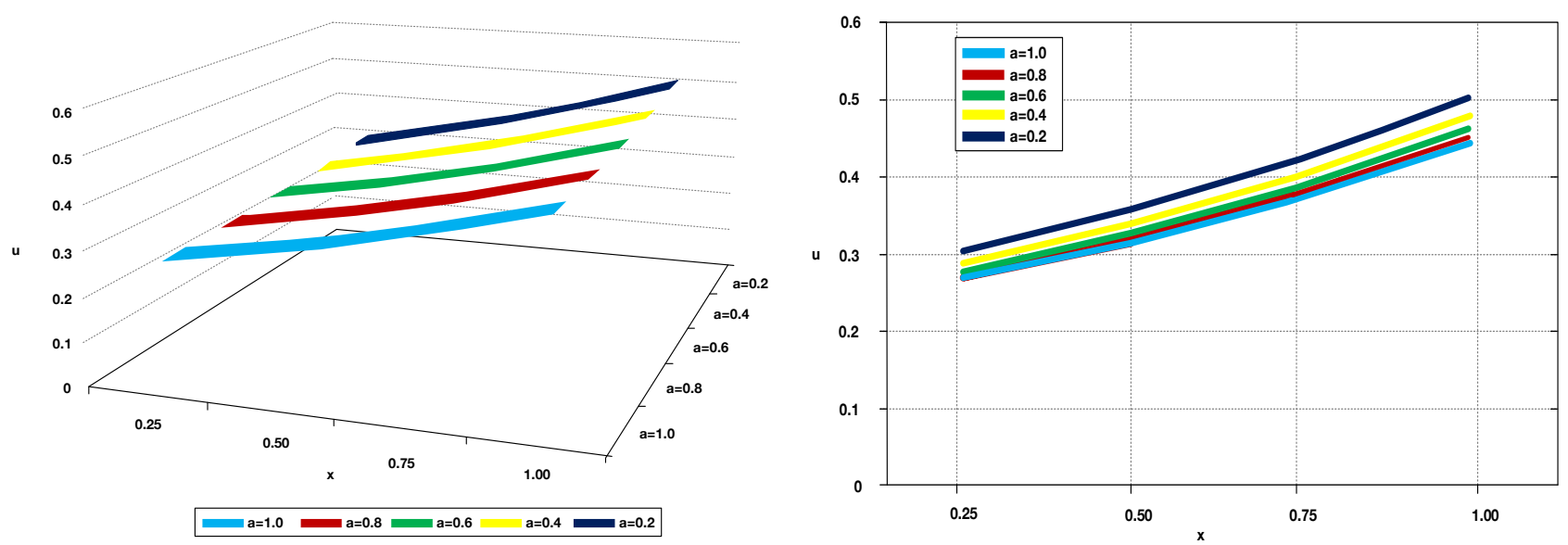

Figure 2: Exact solution of equation (1.1) for an expansion method and $0<\alpha \leqslant 1$

\section{Conclusions}

In this study, we examine the numerical and exact solutions of the time fractional Burgers' equation by using the extended finite difference for numerical solutions, an expansion method and Hopf-Cole transformation for exact solution. Fractional FDMs are useful to solve the nonlinear fractional differential equations by defining suitable fractional differential operators. A stability of the FDM is examined after linearized Burgers' equation by using of Fourier-von Neumann technique. A Burgers' equation is discussed with an example and error estimates obtained for the FDM. Various different situations of fractional order of the numerical solutions are also obtained. The numerical method has been applied to solve a numerical example and results are compared with the corresponding exact solution of time fractional Burgers' equation. Beside this, the behavior of potentials $u$ is examined graphically. These results are presented in a table and Figures 1 and 2 using the package version of Mathematica 11.

\section{References}

[1] D. A. Benson, S. W. Wheatcraft, M. M. Meerschaert, Application of a fractional advection-dispersion equation, Water Resour. Res., 36 (2000), 1403-1412. 1

[2] W. Chen, L.-J. Ye, H.-G. Sun, Fractional diffusion equations by the Kansa method, Comput. Math. Appl., 59 (2010), 1614-1620. 3

[3] P. A. Clarkson, New similarity solutions for the modified Boussinesq equation, J. Phys. A, 22 (1989), 2355-2367. 1

[4] S. A. Elwakil, S. K. El-labany, M. A. Zahran, R. Sabry, Modified extended tanh-function method for solving nonlinear partial differential equations, Phys. Lett. A, 299 (2002), 179-188.

[5] E.-G. Fan, Extended tanh-function method and its applications to nonlinear equations, Phys. Lett. A, 277 (2000), $212-218$. 1

[6] A. Gorguis, A comparison between Cole-Hopf transformation and the decomposition method for solving Burgers' equations, Appl. Math. Comput., 173 (2006), 126-136. 1, 6

[7] S.-M. Guo, Y.-B. Zhou, The extended $\left(\frac{\mathrm{G}^{\prime}}{\mathrm{G}}\right)$-expansion method and its applications to the Whitham-Broer-Kaup-like equations and coupled Hirota-Satsuma KdV equations, Appl. Math. Comput., 215 (2010), 3214-3221. 1

[8] J.-H. He, X.-H. Wu, Exp-function method for nonlinear wave equations, Chaos Solitons Fractals, 30 (2006), 700-708. 1

[9] S. U. Islam, A. J. Khattakand, I. A. Tirmizi, A meshfree method for numerical solution of KdV equation, Eng. Anal. Bound. Elem., 32 (2008), 849-855. 7

[10] F. Liu, P. Zhuang, V. Anh, I. Turner, K. Burrage, Stability and convergence of the difference methods for the space-time fractional advection-diffusion equation, Appl. Math. Comput., 191 (2007), 12-20. 1 
[11] F. Mainardi, M. Raberto, R. Gorenflo, E. Scalas, Fractional calculus and continuous-time finance, II, the waiting-time distribution, Phys. A, 287 (2000), 468-481.

[12] M. M. Meerschaert, C. Tadjeran, Finite difference approximations for fractional advection-dispersion flow equations, J. Comput. Appl. Math., 172 (2004), 65-77. 1

[13] K. S. Miller, B. Ross, An introduction to the fractional calculus and fractional differential equations, A Wiley-Interscience Publication, John Wiley \& Sons, Inc., New York, (1993). 1

[14] Z. M. Odibat, N. T. Shawagfeh, Generalized Taylor's formula, Appl. Math. Comput., 186 (2007), 286-293. 3

[15] K. B. Oldham, J. Spanier, The fractional calculus, Theory and applications of differentiation and integration to arbitrary order, With an annotated chronological bibliography by Bertram Ross, Mathematics in Science and Engineering, Academic Press [A subsidiary of Harcourt Brace Jovanovich, Publishers], New York-London, (2006). 1

[16] E. J. Parkes, B. R. Duffy, An automated tanh-function method for finding solitary wave solutions to non-linear evolution equations, Comput. Phys. Commun., 98 (1996), 288-300. 1

[17] I. Podlubny, Fractional differential equations, An introduction to fractional derivatives, fractional differential equations, to methods of their solution and some of their applications, Mathematics in Science and Engineering, Academic Press, Inc., San Diego, CA, (1999). 1

[18] E. Scalas, R. Gorenflo, F. Mainardi, Fractional calculus and continuous-time finance, Phys. A, 284 (2000), 376-384. 1

[19] E. Sousa, Finite difference approximations for a fractional advection diffusion problem, J. Comput. Phys., 228 (2009), 4038-4054.

[20] L.-J. Su, W.-Q. Wang, Q.-Y. Xu, Finite difference methods for fractional dispersion equations, Appl. Math. Comput., 216 (2010), 3329-3334.

[21] L.-J. Su, W.-Q. Wang, Z.-X. Yang, Finite difference approximations for the fractional advection-diffusion equation, Phys. Lett. A, 373 (2009), 4405-4408. 1

[22] M.-L. Wang, X.-Z. Li, J.-L. Zhang, The $\left(\frac{\mathrm{G}^{\prime}}{\mathrm{G}}\right)$-expansion method and travelling wave solutions of nonlinear evolution equations in mathematical physics, Phys. Lett. A, 372 (2008), 417-423. 1

[23] A. M. Wazwaz, The tanh method: solitons and periodic solutions for the Dodd-Bullough-Mikhailov and the Tzitzeica-DoddBullough equations, Chaos Solitons Fractals, 25 (2005), 55-63. 1

[24] A. Yokus, Solutions of some nonlinear partial differential equations and comparison of their solutions, Ph.D. Thesis, Firat University, Elazig, Turkey, (2011). 1

[25] S. B. Yuste, Weighted average finite difference methods for fractional diffusion equations, J. Comput. Phys. , 216 (2006), 264-274. 1

[26] G. M. Zaslavsky, Chaos, fractional kinetics, and anomalous transport, Phys. Rep., 371 (2002), 461-580. 1

[27] X.-D. Zheng, Y. Chen, H.-Q. Zhang, Generalized extended tanh-function method and its application to $(1+1)-$ dimensional dispersive long wave equation, Phys. Lett. A, 311 (2003), 145-157. 1

[28] P. Zhuang, F. Liu, V. Anh, I. Turner, New solution and analytical techniques of the implicit numerical method for the anomalous subdiffusion equation, SIAM J. Numer. Anal., 46 (2008), 1079-1095. 1 